\title{
O CENTENÁRIO DA TEORIA DE BOHR
}

João Pedro Braga e Carlos A. L. Filgueiras*

Departamento de Química, Universidade Federal de Minas Gerais, 31270-901 Belo Horizonte - MG, Brasil

Recebido em 24/6/13; aceito em 1/7/13; publicado na web em 19/7/13

\begin{abstract}
THE CENTENNIAL OF BOHR'S THEORY. The year 2013 marks the centennial of that wondrous year in which Niels Bohr proposed a novel theory about the constitution of atoms and small molecules after which the way we regard atoms and their behaviour began to be drastically altered. Bohr drew on several sources for his original description of the atoms, most importantly on spectroscopy and Balmer's equation thereof, the new quantum hypothesis advanced a few years earlier by Planck, and the planetary atom proposed by Rutherford. Although Bohr's ideas were to be eventually overtaken by the advent of quantum mechanics, his theory was the basis of a new thinking about atoms and molecules which constitutes an invaluable asset in the development of science ever
\end{abstract} since.

Keywords: Niels Bohr; Bohr's Theory of atoms; the constitution of atoms and molecules.

\section{INTRODUÇÃO}

No ano de 2013 completa um século aquele annus mirabilis em que o físico dinamarquês Niels Bohr publicou sua teoria sobre a constituição dos átomos. Essa foi a primeira explicação da estrutura atômica a lançar mão de pressupostos quânticos, criando uma clivagem com as teorias clássicas precedentes e abrindo o caminho para o entendimento moderno do átomo. Ao publicar o primeiro de seus artigos de 1913 que subitamente o lançaram à vanguarda da ciência mundial, Bohr era um jovem de 28 anos que só havia até então publicado três artigos de seu tempo de estudante. ${ }^{1}$

A fórmula do suíço Johann Balmer, de 1885, que explicava numericamente, de forma simples, os valores de absorção ou emissão de energia radiante no átomo de hidrogênio, o conceito de quantum de energia proposto por Max Planck em 1900, e o modelo planetário do átomo de Ernest Rutherford, de 1911, foram os três alicerces fundamentais no desenvolvimento da teoria do átomo de Niels Bohr, elaborada e publicada em 1913.

\section{AS PRIMEIRAS DÉCADAS DA VIDA DE BOHR}

Niels Henrick David Bohr (1885-1962) nasceu em Copenhagen, filho de um professor de Fisiologia na Universidade de Copenhagen, Christian Bohr (1855-1911). Ele teve uma irmã, Jenny (1883-1933), e um irmão, Harald (1887-1951), que se tornou um importante matemático. A Figura 1 mostra os irmãos Bohr e seu pai em 1906.

A educação de Bohr se deu na Universidade de Copenhagen, onde ele obteve sua formação científica, defendendo uma tese de doutorado em 1911 intitulada "Estudos sobre a Teoria Eletrônica dos Metais", cujo tema já tinha sido parcialmente esboçado no mestrado, finalizado em 1909. Em 1912 Niels Bohr se casou com Margrethe Nørlund, com quem teve seis filhos. Um deles, Aage Niels Bohr (1922-2009), ganhou o Prêmio Nobel de Física em 1975, assim como seu pai em 1922. ${ }^{2}$

Bohr viria a fundar o Instituto de Física Teórica da Universidade de Copenhagen em 1920, o qual viria a ter seu nome mudado para Instituto Niels Bohr.

Bohr tinha um espírito inquieto e sempre se interessou por toda a ciência, e não apenas a Física, o que é bem ilustrado pelo seguinte episódio.

\footnotetext{
*e-mail: calfilgueiras@gmail.com
}

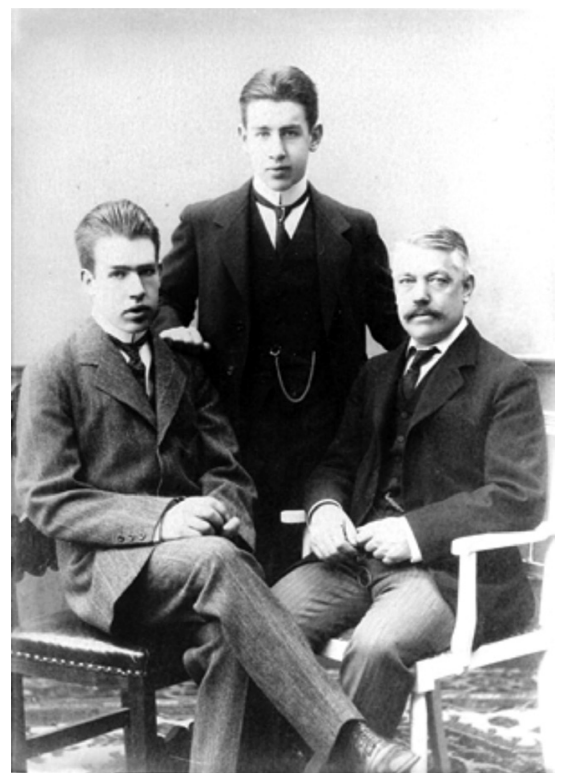

Figura 1. Niels Bohr, seu irmão, o matemático Harald Bohr, e o pai de ambos, Christian Bohr, professor de Fisiologia na Universidade de Copenhagen, em foto de 1906. Foto cedida gentilmente pelo Arquivo Bohr, Copenhagen

O trabalho precoce de Henry Moseley (1887-1915) em Manchester com Ernest Rutherford (1871-1937) mostrou que ao bombardear-se um alvo metálico com raios catódicos, o alvo emitia raios X com uma frequência característica do metal. Os quadrados das frequências dos raios $\mathrm{X}$ emitidos dão uma sequência numérica, chamada série de Moseley. Esta sequência é hoje a série de números atômicos, que vieram a substituir as massas atômicas no ordenamento dos elementos na Classificação Periódica. O trabalho de Moseley cobriu desde o alumínio $(\mathrm{Z}=13)$ ao ouro $(\mathrm{Z}=79)$, prevendo a existência dos elementos 43, 61, 72, 75, 85, 87 e 91 .

Com base em seu conhecimento da estrutura atômica dos elementos, Niels Bohr previu que o elemento 72 seria quimicamente diferente do lutécio, ou seja, não seria uma nova terra rara, mas sim um elemento análogo ao zircônio. O químico francês Georges Urbain (1872-1938) havia tentado por muito tempo, infrutiferamente, encontrar o elemento 72 em resíduos de terras raras. A partir de sua teoria quântica, Bohr havia construído uma Tabela Periódica com 
os grupos dispostos horizontalmente e os períodos na vertical, ao contrário das tabelas modernas. Com o conhecimento de sua teoria de constituição dos átomos, ele opinou que o novo elemento deveria ser quadrivalente, e não trivalente, por isso não deveria ser uma nova terra rara, e sim um elemento do grupo do zircônio. Ele então aconselhou o húngaro George de Hevesy (1889-1966) a procurar o elemento 72 em minerais de zircônio, o que de Hevesy e o holandês Dirck Coster (1889-1950) fizeram em Copenhagen, isolando o novo elemento em 1923. Ele ganhou o nome de Háfnio em homenagem à cidade de Copenhagen, cujo nome latinizado é Hafnia. ${ }^{3,4}$ Hoje, outro elemento, o de número atômico 107, é chamado bóhrio em homenagem a Niels Bohr.

Bohr gostava de usar as palavras da forma mais precisa possível, como os termos de uma fórmula matemática. Quando jovem, após seu doutorado em Copenhagen, foi a Cambridge em 1911 estudar com Joseph John Thomson (1856-1940), o famoso Cavendish Professor of Physics daquela universidade britânica. Bohr tinha alguma dificuldade no uso da língua inglesa. Ninguém o entendia quando falava de uma propriedade do elétron que ele chamava de "load". Só algum tempo mais tarde ele próprio descobriu que a palavra inglesa para carga era "charge". Para corrigir seus erros, comprou um dicionário e a obra completa de Charles Dickens, lendo tudo e anotando as palavras que não conhecia, com ajuda do dicionário. ${ }^{5}$ Seu inglês defeituoso fez com que ele não causasse boa impressão em J. J. Thomson, levando-o, em parte, a decidir mudar de instituição e trabalhar com Ernest Rutherford na Universidade Victoria, em Manchester. Sua ligação com Rutherford mostrou-se providencial. Ele foi um dos primeiros físicos a perceber a importância do conceito de quantum de Max Planck, introduzido em 1900, o que lhe foi de suma importância quando se propôs a entender teoricamente o modelo atômico planetário de Rutherford, que descartava o modelo atômico anterior de J. J. Thomson, comumente chamado de pudim de passas, que supunha as partes positivas e os elétrons em contacto íntimo.

Entre as muitas histórias anedóticas a respeito de Bohr, é interessante relatar a seguinte. Em 1916, criou-se para ele uma cadeira de Física Teórica na Universidade de Copenhagen. De acordo com a praxe, os novos catedráticos eram apresentados ao Rei da Dinamarca, Christian X. Este, ao cumprimentar Bohr, disse como estava feliz de encontrar um jogador de futebol tão famoso. Bohr retrucou que Sua Majestade deveria estar pensando em seu irmão Harald, que tinha jogado pela Dinamarca nos Jogos Olímpicos de 1908. O Rei ficou irritado de ser desmentido em público, o que fez Bohr dizer que ele também jogava como goleiro, mas não era o famoso jogador a que o Rei se referira. Ao ouvir isto o Rei exclamou: "A audiência acabou!"6

\section{O MISTÉRIO DA DESCONTINUIDADE NAS RAIAS ESPECTROSCÓPICAS}

É interessante que a espectroscopia atômica estava bem menos ligada ao resto da física na segunda metade do século XIX que hoje, embora fosse tão fundamental para os químicos, que a usaram para descobrir vários elementos novos, desde que Robert Bunsen e Gustav Kirchhoff se uniram para inventar o espectroscópio em $1859 .{ }^{7}$ A partir de experimentos realizados com o sódio, Kirchhoff publicou em 1859 suas três leis da espectroscopia:

1. Um corpo incandescente emite um espectro contínuo;

2. Um corpo excitado, como o sódio em forma de vapor, emite um espectro discreto, formado por linhas brilhantes;

3. Quando a luz branca passa através do vapor de um elemento, o espectro resultante apresenta linhas escuras de absorção, nas frequências em que o vapor emite linhas brilhantes. ${ }^{8}$

Usando o novo espectroscópio por eles inventado, Bunsen e Kirchhoff descobriram dois novos elementos, o césio, em 1860, e o rubídio, em 1861. O césio foi descoberto em águas minerais de Dürkheim, e o rubídio em resíduos alcalinos extraídos do mineral lepidolita. O césio apresenta linhas na faixa do azul, donde seu nome (cesius era a cor do firmamento em latim), ao passo que o rubídio tem suas linhas no vermelho, por isso o nome derivado de rubi. ${ }^{8}$ Vários outros elementos foram descobertos sucessivamente pela espectroscopia atômica, mas o caso mais espetacular aconteceu em 1868. O astrônomo francês Pierre Janssen (1824-1907) foi naquele ano à Índia para observar um eclipse total do sol e realizar o primeiro estudo espectroscópico de sua cromosfera. Ele percebeu no espectro solar uma linha na faixa do amarelo $\left(\mathrm{D}_{3}\right)$, que não coincidia com a linha D do sódio, e que ele era incapaz de repetir no laboratório. $\mathrm{O}$ astrônomo inglês Sir Norman Lockyer (1836-1920) interessou-se pelo assunto e mostrou que a nova linha era distinta das linhas de qualquer elemento conhecido, atribuindo-a a um novo elemento desconhecido na terra, e chamando-o hélio, do nome do deus grego do sol. Finalmente, em 1895, Sir William Ramsay (1852-1916) conseguiu mostrar que quando o mineral de urânio denominado cleveíta é tratado com ácidos fortes, libera uma mistura de gases, compreendendo o nitrogênio, o argônio e um outro gás com linhas espectrais diferentes. Enviando uma ampola com amostra deste gás para Lockyer e William Crookes (1832-1919), que dispunham de melhores espectroscópios, foi possível provar sem qualquer dúvida que se tratava do mesmo elemento descoberto 27 anos antes no sol. ${ }^{9}$ Esta descoberta de um novo elemento no sol quase três décadas antes de o mesmo ser descoberto na terra mostrou a imensa importância da espectroscopia na análise química e na identificação das propriedades individuais de cada elemento.

Um grande problema que permanecia insolúvel, contudo, era como relacionar entre si os diferentes valores das frequências das raias espectrais de cada elemento. Várias tentativas foram feitas, supondo algum modelo vibracional harmônico ou anarmônico, mas todas essas tentativas levaram ao fracasso.

\section{A EQUAÇÃO DE BALMER}

O problema da relação entre as frequências espectrais foi muito discutido por físicos, químicos e astrônomos, mas foi um matemático, o suíço Johann Jakob Balmer (1825-1898), que conseguiu resolvê-lo. Balmer obteve um doutorado em matemática e passou a vida como professor dessa disciplina numa escola secundária para moças em Basiléia. Como já se disse aqui, os físicos tentavam achar uma relação para as linhas espectrais baseando-se numa analogia mecânico-acústica, e buscavam expressões harmônicas simples que explicassem essas relações. Talvez por não ser físico e sim matemático, isto é, por não partir de posições preconcebidas, Balmer chegou em 1885 à equação que hoje traz seu nome e que expressa perfeitamente a relação numérica das linhas do espectro conhecido à época para o hidrogênio. A equação de Balmer, que todo estudante de química geral aprende, é modernamente formulada como:

$$
\frac{1}{\lambda}=\mathrm{R}\left(\frac{1}{2^{2}}-\frac{1}{n^{2}}\right)
$$

em que $\lambda=$ comprimento de onda correspondente à linha espectral (em cm); R = constante, chamada hoje de constante de Rydberg, que provou ser igual a $109677 \mathrm{~cm}^{-1} ; \mathrm{n}=3,4,5,6, \ldots$

A equação de Balmer descreve adequadamente os valores das frequências dos espectros de emissão ou absorção do hidrogênio na região visível, mas pode ser modificada para incluir também as outras regiões espectrais. Para outros elementos podem ser usadas equações análogas, mas a precisão é tanto menor quanto mais pesado for o elemento. Os comprimentos de onda (ou as energias) correspondentes 
às linhas que resultam da absorção ou emissão de energia estão relacionados entre si por números inteiros (n é a variável independente da equação, com valores dados por $3,4,5,6 \ldots$... . Consequentemente, os ganhos ou perdas de energia nos átomos são discretos e também guardam uma relação de números inteiros. ${ }^{7}$

\section{A DESCONTINUIDADE QUÂNTICA}

A teoria quântica de Max Planck (1858-1947) foi um dos pilares da descrição que Bohr daria para o átomo em seu modelo teórico de 1913. Antes de falar de Planck, é preciso, contudo, mostrar a situação a respeito de um grande problema do final do século XIX, qual seja, a questão da radiação do corpo negro, um objeto hipotético capaz de absorver ou emitir radiação sem quaisquer perdas. Várias formulações surgiram, uma baseada na Termodinâmica clássica, a Lei de Rayleigh-Jeans, bastante discrepante da realidade à medida que diminuía o comprimento de onda da radiação, e a outra, a Lei de Wien, uma aproximação bastante de acordo com os dados experimentais.

\section{LEIS DE RAYLEIGH-JEANS (1900-1905) E DE WIEN (1893)}

A Lei de Rayleigh-Jeans, derivada de pressupostos da termodinâmica clássica, leva a uma expressão analítica que mostra, em representação gráfica, a intensidade da radiação contra o comprimento de onda para cada temperatura considerada. ${ }^{10} \mathrm{O}$ gráfico de intensidade de radiação contra comprimento de onda está mostrado na Figura 2.

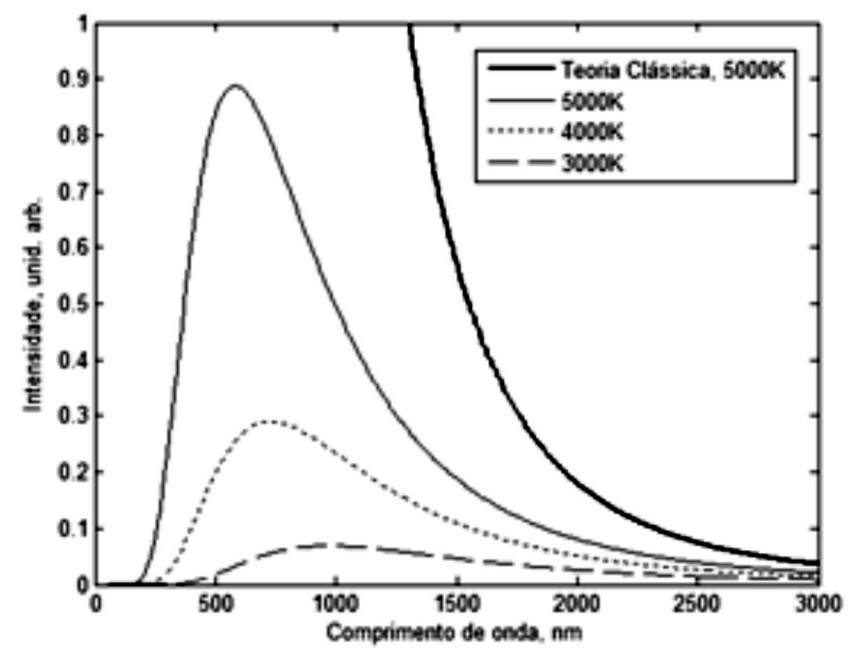

Figura 2. Intensidade de radiação contra comprimento de onda (cortesia de Nelson H.T. Lemes)

$$
B_{\lambda}(T)=\frac{2 k T\left(\frac{c}{\lambda}\right)^{2}}{c^{2}} \times \frac{c}{\lambda^{2}}=\frac{2 c k T}{\lambda^{4}}
$$

$B_{\lambda}(T)=$ intensidade da radiação de comprimento de onda $\lambda$ à temperatura $\mathrm{T}$

$\mathrm{k}=$ constante de Boltzmann

$\mathrm{T}=$ temperatura em kelvins

$\mathrm{c}=$ velocidade da luz

Pela Lei de Rayleigh-Jeans, para pequenos comprimentos de onda a intensidade da radiação se aproximaria de infinito. Esta é a chamada catástrofe do ultravioleta. O universo deveria estar inundado de uma quantidade infinita de radiação de alta energia, isto é, de pequenos comprimentos de onda. Como isso não ocorre, a lei de Rayleigh-Jeans, obtida de suposições clássicas, não descreve corretamente o fenômeno.
A Lei de Wien (1893), ${ }^{11}$ é uma aproximação que satisfaz os dados experimentais:

$$
\lambda_{\max }=b / T, \text { em que: }
$$

$\lambda_{\max }=$ comprimento de onda do pico de uma curva de radiação

$\mathrm{T}=$ temperatura em kelvins

$\mathrm{b}=$ constante de Wien

\section{A HIPÓTESE QUÂNTICA DE PLANCK}

Supondo que a Lei de Wien dá uma boa descrição da radiação do corpo negro, sobretudo para comprimentos de onda menores, Planck se dispôs a reexaminar as premissas usadas por Wien em sua derivação.

Sem dar aqui a derivação de Planck, que estenderia demasiadamente este tópico, basta dizer que no limite de valores de $\lambda$ maiores, a Lei de Planck coincide com a Lei de Rayleigh-Jeans, ao passo que no limite de valores de $\lambda$ menores ela tende à aproximação de Wien. Planck supôs um experimento imaginário em que para um oscilador com uma determinada frequência, sua energia só poderia ser dada em múltiplos integrais de $\mathrm{E}=\mathrm{h} v$, em que hé uma constante conhecida hoje como constante de Planck. Quando ocorre variação de energia nos osciladores atômicos, isto é, emissão ou absorção de energia, esta variação só se dá em "pacotes" discretos, e não continuamente. A fórmula $\mathrm{E}=\mathrm{h} v$ corresponde a dizer que as quantidades discretas de energia que um oscilador pode emitir ou absorver são diretamente proporcionais a sua frequência. Os osciladores de baixa frequência só aceitam energia em pacotes pequenos, ao passo que os osciladores de alta frequência aceitam pacotes grandes. Há uma distribuição estatística dos osciladores, eliminando a catástrofe do ultravioleta: em temperaturas mais altas, as oscilações atômicas se tornam mais energéticas e a radiação emitida desloca-se para frequências mais altas. O contrário ocorre ao diminuir a temperatura. Cada pacote de energia é denominado um "quantum". Os valores discretos da energia de um oscilador são dados como $\mathrm{E}=\mathrm{nhv}$, em que $\mathrm{n}=1,2,3,4, \ldots{ }^{12}$

Assim como a fórmula de Balmer, a teorização de Planck era derivada de dados empíricos. Não se sabia nem a razão de as frequências das raias do espectro atômico seguirem a equação de Balmer, nem tampouco a origem da hipótese quântica de Planck.

\section{O ÁTOMO PLANETÁRIO DE RUTHERFORD}

O primeiro emprego acadêmico do físico neo-zelandês Ernest Rutherford (1871-1937) foi como professor na Universidade McGill, em Montreal, no Canadá. Ele havia sido recomendado por seu mentor científico, J. J. Thomson. Rutherford viera da Nova Zelândia em 1895, onde cursara a Universidade em Wellington, para trabalhar em Cambridge com J. J. Thomson, diretor do Laboratório Cavendish de Física, e futuro descobridor do elétron em 1897. Em McGill, onde permaneceu até 1907, Rutherford se destacou por seus experimentos com o decaimento de substâncias radioativas, o que lhe valeu o Prêmio Nobel de Química em 1908. A concessão do prêmio não deixa de ser irônica, em vista de sua baixa estima pela Química. Aliás, ele disse a respeito do Prêmio Nobel que lhe foi conferido que ele tinha observado em seu trabalho sobre o decaimento radioativo muitas transformações, mas nenhuma tão rápida como a sua, de físico em químico. ${ }^{13}$ Entre 1907 e 1919 ele foi professor em Manchester, de onde retornou a Cambridge, como sucessor de Thomson, até sua morte em 1937. Foi depois de ganhar o Prêmio Nobel que ele fez sua descoberta mais conhecida, a do átomo nuclear, em 1911. Este é um caso singular na história da ciência, de alguém que tenha feito seu 
trabalho mais importante depois de já ter recebido o Prêmio Nobel em sua área de pesquisa. Trabalhando em Manchester em colaboração com o físico alemão Hans Geiger (1882-1945) e o então estudante de graduação Ernest Marsden (1889-1970), Rutherford realizou o famoso experimento de bombardeamento de uma delgada folha de ouro com partículas alfa. Desse experimento, amplamente relatado na literatura e em livros-textos, resultou que a imensa maioria das partículas alfa passava pelo metal sem sofrer qualquer deflexão. Uma primeira conclusão foi que o átomo tem um grande volume vazio. Com relação àquelas que sofriam deflexão, foi possível mostrar que esta deflexão resultava de interações com uma parte maciça e carregada positivamente no átomo, que tinha um volume de apenas $10^{-4}$ vezes o volume do átomo. Consequentemente, os modelos atômicos anteriores, inclusive o de Thomson, perderam a validade. A partir daí, Rutherford propôs seu novo modelo, o do átomo nuclear, com os elétrons dispostos em torno de um núcleo positivo. ${ }^{14} \mathrm{Em}$ seu modelo ele supôs uma situação análoga à do sistema solar, que não agradou em nada a J. J. Thomson, pois o modelo tinha dificuldades incontornáveis para a física da época. Num modelo planetário, o número de órbitas possíveis é infinito. Ademais, ao sofrer rotação em torno do núcleo, o elétron devia irradiar energia, como ocorre com cargas em movimento. Consequentemente, a energia total do elétron devia diminuir continuamente e eventualmente ele seguiria um movimento em espiral e cairia no núcleo. Em outras palavras, o átomo planetário seria altamente instável. A Figura 3 mostra o jovem Bohr aos 26 anos de idade, em 1911, dois anos antes de publicar sua teoria sobre o átomo e as pequenas moléculas.

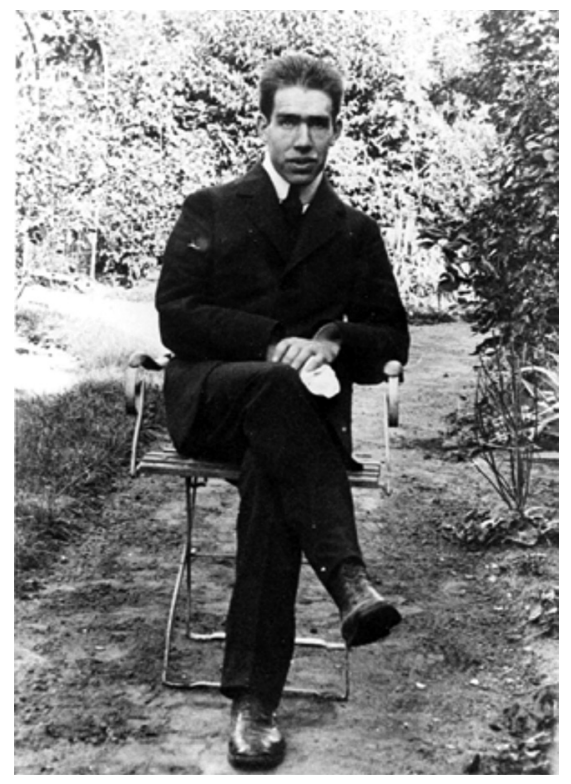

Figura 3. Niels Bohr em 1911, dois anos antes de publicar os três artigos sobre a constituição dos átomos e moléculas. Foto cedida gentilmente pelo Arquivo Bohr, Copenhagen

\section{O MODELO DE BOHR}

Foi no início do ano de 1913 que Bohr teve sua atenção atraída pela fórmula de Balmer, lendo sobre ela no livro de Stark, Prinzipien der Atomdynamik, de $1911 .{ }^{15}$ Neste mesmo ano de 1913, ele daria ao mundo sua mais importante contribuição científica, conhecida como o modelo atômico de Bohr.

O trabalho de Bohr sobre a constituição do átomo foi publicado em três artigos, todos saídos à luz no ano de $1913 .{ }^{16-18}$ Esses três artigos seminais, os mais importantes de toda a carreira de Bohr, estão disponíveis em tradução para o português num único volume, sob o título geral de Sobre a Constituição de Átomos e Moléculas, ${ }^{19}$ e os subtítulos:

Parte I - Ligação de Elétrons por Núcleos Positivos;

Parte II - Sistemas que Contêm um só Núcleo;

Parte III - Sistemas que Contêm Vários Núcleos.

Esse conjunto de três artigos inaugurou a ciência moderna do átomo.

O primeiro deles, sobre a constituição do átomo de hidrogênio, introduziu o conceito de estados estacionários, órbitas eletrônicas em que os elétrons não absorveriam ou emitiriam energia, o que aconteceria quando passassem de um estado para outro. A partir daí Bohr pôde chegar a uma fórmula idêntica à equação de Balmer. Até então Bohr só tinha publicado três artigos, todos de seu tempo de estudante. ${ }^{19}$ Esse primeiro artigo de 1913, dedicado a átomos com um elétron, foi sucedido por um segundo sobre átomos multieletrônicos. O terceiro, como diz o próprio título, trata de sistemas de mais de um núcleo, isto é, moléculas.

Como ressalta o historiador da ciência Helge Kragh, o tratamento das moléculas por Bohr ficou historicamente relegado a um plano inferior, com muito pouca ênfase comparado a seu trabalho sobre os átomos individuais. ${ }^{20}$ Da mesma forma, embora Bohr tenha tido bastante sucesso entre os químicos no que respeita à aplicação de suas ideias na construção da Tabela Periódica, usando o Princípio do Aufbau, que todo estudante aprende hoje em dia, em outros aspectos sua teoria quântica dos átomos foi largamente ignorada pelos químicos até o final dos anos 20. Isto porque estes preferiam muito mais modelos estáticos do tipo daqueles de Gilbert Lewis (1875-1946), como se vê em seu livro clássico Valence and the Structure of Atoms and Molecules, de 1923, ${ }^{21}$ em que os elétrons nos átomos se disporiam segundo os vértices de um cubo, bem diferente do modelo dinâmico de Bohr, com os elétrons movendo-se rapidamente em suas órbitas. Nos modelos químicos, com os elétrons ocupando posições fixas nos vértices de um cubo, era muito mais fácil imaginar as ligações químicas formadas por compartilhamento eletrônico e a geometria de moléculas que tivessem, por exemplo, um carbono tetraédrico. No modelo físico de Bohr, isto simplesmente não era possível imaginar. ${ }^{22}$ Como bem disse Helge Kragh, havia uma dicotomia intransponível entre a forma de pensar química e aquela dos físicos. ${ }^{20}$ Só muito mais tarde, após o aparecimento da mecânica quântica, que veio substituir o átomo de Bohr pelo modelo atual, e com o advento da química quântica a partir de 1930, em que os fenômenos atômicos e moleculares passaram a ser vistos sob uma óptica distinta daquela dos físicos, isto é, sob um ponto de vista químico, é que foi possível uma reaproximação dos dois campos. Esse foi o trabalho de vários químicos notáveis, entre os quais ressalta a figura de Linus Pauling $(1901-1994){ }^{23}$

\section{AGRADECIMENTO}

Os autores agradecem o suporte do CNPq.

\section{REFERÊNCIAS}

1. Pais, A.; Niels Bohr's Times, in Physics, Philosophy and Polity, Clarendon Press: Oxford, 1991, p. 146.

2. Ref. 1, p. 107.

3. Ihde, A. J.; The Development of Modern Chemistry, Harper \& Row: New York, 1964, p. 589.

4. Weeks, M. E; Leicester, H. M.; Discovery of the elements, $7^{\text {th }}$ ed., J. Chem. Educ. Press: Easton, 1968, p. 816-822.

5. Cline, B. L.; Men Who Made a new Physics - Physicists and the Quantum Theory, The New American Library: New York, 1969, p. 80.

6. Ref. 1, p. 166-67. 
7. Filgueiras, C. A. L., Química Nova na Escola 1996, 3, 22.

8. Ref. 3, p. 235.

9. Ref. 4, p. 757-764.

10. a) Braga, J. P.; Quim. Nova 2001, 24, 693; b) Braga, J. P.; Fundamentos da Química Quântica, Editora da Universidade Federal de Viçosa, 2007, p. 9 .

11. Hund, F. H.; The History of Quantum Theory, Harrap: London, 1974, p. 23.

12. Guillemin, V.; The Story of Quantum Mechanics, Scribner's: New York, 1968, p. 50-52.

13. Ref. 5, p. 27

14. Ref. 1, p. 121-129.

15. Ref. 1, p. 143-144

16. Bohr, N.; Phil. Mag. 1913, 26, 1.
17. Bohr, N., Phil. Mag. 1913, 26, 476.

18. Bohr, N.; Phil Mag. 1913, 26, 867.

19. Bohr, N; Sobre a Constituição de Átomos e Moléculas, Textos Fundamentais da Física Moderna, $2^{\mathrm{a}}$ ed., Fundação Calouste Gulbenkian: Lisboa, 1979. No original inglês, não consta o subtítulo da primeira parte, dado na versão portuguesa aqui citada.

20. Kragh, H.; Physics Today; http://www.physicstoday.org/resource/1/ phtoad/v66/i5/p36_s1 ?bypassSSO=1, acessada em Julho 2013.

21. Lewis, G. N.; Valence and the Structure of Atoms and Molecules, Dover: New York, 1966, p. 29.

22. Kasha, M., Pure Appl. Chem. 1990, 62, 1615.

23. Gavroglu, K.; Simões, A.; Neither Physics nor Chemistry - A History of Quantum Chemistry, MIT Press: Cambridge, 2012. 